\section{Enhanced stimulated Brillouin scattering threshold through phase control of multitone phase modulation}

\author{
John C. Mauro, Srikanth Raghavan, and A. Boh Ruffin \\ Corning Incorporated, Science and Technology Division, \\ Corning, New York 14831 \\ E-mail:mauroj@corning.com
}

\begin{abstract}
We show that controlling the phase difference among the various tones of a multitone phase modulator can substantially increase the stimulated Brillouin scattering (SBS) threshold during optical fiber transmission. We present detailed modeling and experimental validation of the effect. This result should be especially useful for optical networks requiring high signal launch power. (C) 2010 Society of Photo-Optical Instrumentation Engineers. [DOI: 10.1117/1.3496300]
\end{abstract}

Subject terms: stimulated Brillouin scattering; phase modulation; fiber optic communication systems.

Paper 100597LR received Jul. 23, 2010; revised manuscript received Sep. 2, 2010; accepted for publication Sep. 7, 2010; published online Oct. 26, 2010.

High signal launch powers are often necessary in fiber optic networks to meet signal-to-noise ratio and receiver sensitivity requirements. ${ }^{1}$ One of the drawbacks of high launch powers is the nonlinear phenomenon of stimulated Brillouin scattering (SBS), which can be suppressed through use of advanced transmission fiber, ${ }^{1,2}$ through proper choice of parameters in single-frequency amplifier design, ${ }^{3-5}$ or through signal broadening at the transmitter. ${ }^{6-10}$ Two standard methods have been proposed to suppress SBS through transmitter design: 1. dithering of the optical frequency of the laser transmitter, and 2. imparting a sinusoidal phase modulation to the optical carrier. ${ }^{6-10}$ Essentially, both these methods involve broadening the optical spectrum, thereby increasing the SBS threshold power. ${ }^{9}$ Whereas these works have generally focused on phase modulation using a single sinusoidal drive (hereafter referred to as single-tone phase modulation) and have largely ignored the phase relationships between dithering and phase modulation tones, we focus our attention on multitone phase modulation and show that the phase relationship among the various tones can impact the resulting SBS threshold by several decibels. Incorporation of a phaselocking mechanism to control the phase relationship among the various phase modulation tones may provide a significant advantage in high-power optical networks.

We begin by considering a general optical carrier with $N$ phase modulation tones,

$E(t)=E_{0}(t) \prod_{n=1}^{N} \exp \left[i A_{n} \cos \left(2 \pi f_{n} t+\phi_{n}\right)\right]$,

0091-3286/2010/\$25.00 @ 2010 SPIE where $E_{0}(t)$ is the slowly varying envelope, and $A_{n}, f_{n}$, and $\phi_{n}$ are the amplitude, frequency, and phase of the $n$th tone, respectively. Since the SBS threshold depends on the distribution of power in the optical spectrum, we must first calculate the optical power spectral density (PSD) and validate against experiment. We calculate the PSD by assuming a $\mathrm{cw}$ input and expanding Eq. (1) in a Fourier Bessel series. The result is a series of Dirac delta functions with weighting coefficients that depend on $A_{n}$ and $\phi_{n}$. Finally, we apply a Gaussian line shape to each peak, where the line width is obtained based on experimental measurement. In Fig. 1(a), we show a comparison between the optical spectrum from the experiment and our modeling for the case of a single phase modulation tone at $f_{1}=3.0 \mathrm{GHz}$ and $A_{1}=5.71 \mathrm{rad}$. The input signal $E_{0}(t)$ has a measured full width at half maximum (FWHM) line width of $1.25 \mathrm{GHz}$. In Fig. 1(b), we show a comparison between the experimental and theoretical optical spectra for the case of three-tone phase modulation with $f_{1}=3.0 \mathrm{GHz}$, $A_{1}=5.71 \mathrm{rad}, f_{2}=2.75 \mathrm{GHz}, A_{2}=4.92 \mathrm{rad}, f_{3}=2.5 \mathrm{GHz}$, and $A_{3}=4.72 \mathrm{rad}$. The relative phases of the three tones are $\phi_{1}=3.05 \mathrm{rad}, \phi_{2}=3.67 \mathrm{rad}$, and $\phi_{3}=0$. We achieve excellent agreement between theory and experiment for both the single- and multitone cases.

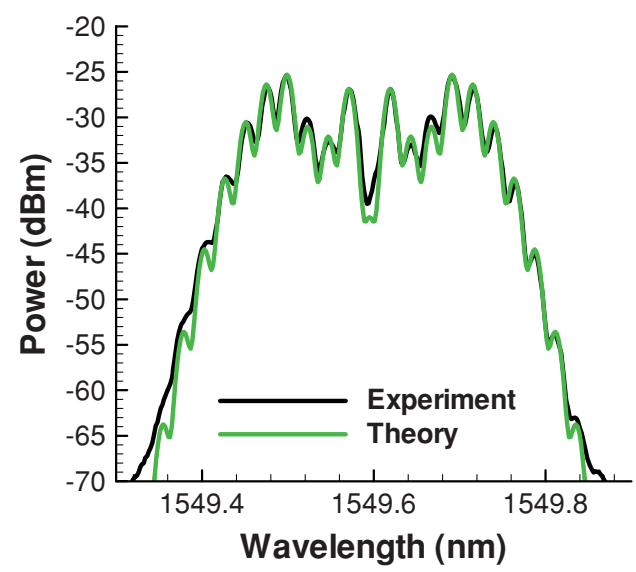

(a)

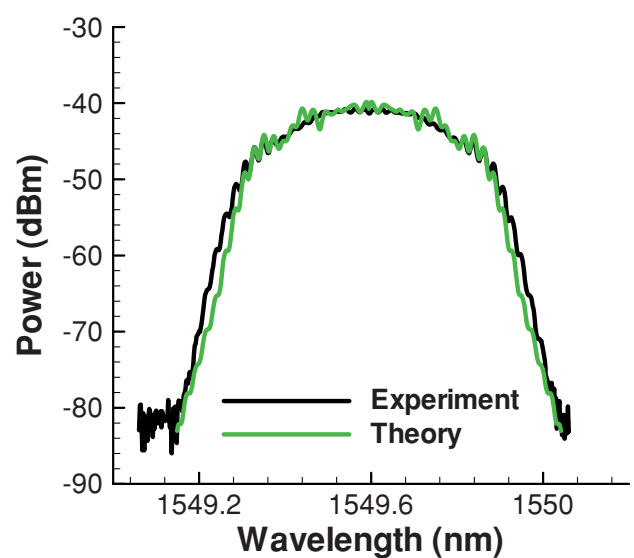

(b)

Fig. 1 Validation of simulated optical spectrum versus experiment for the cases of (a) single-tone phase modulation at $3 \mathrm{GHz}$ and (b) three-tone phase modulation with frequencies of 2.5, 2.75, and 3.0 $\mathrm{GHz}$. 


\section{OE Letters}

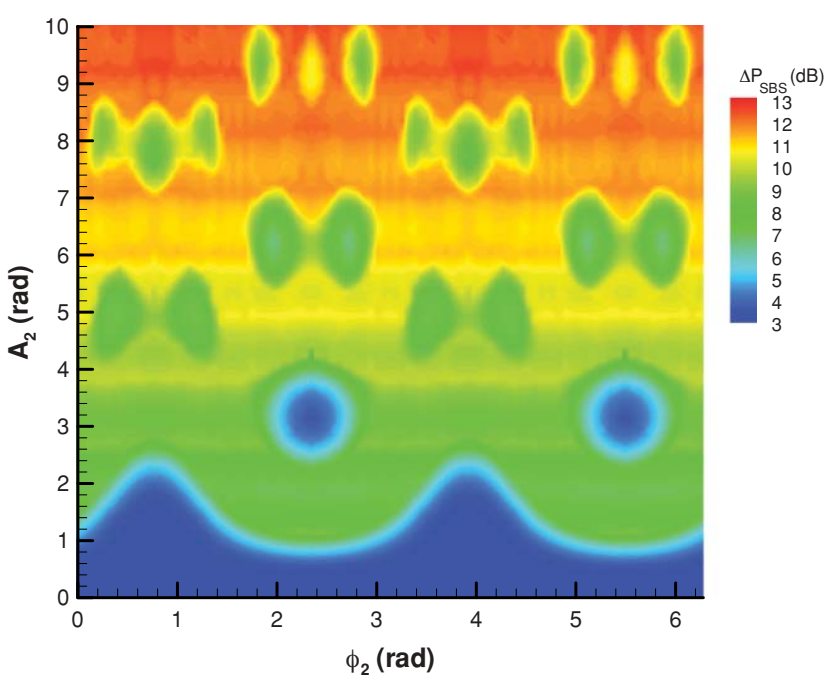

(a)

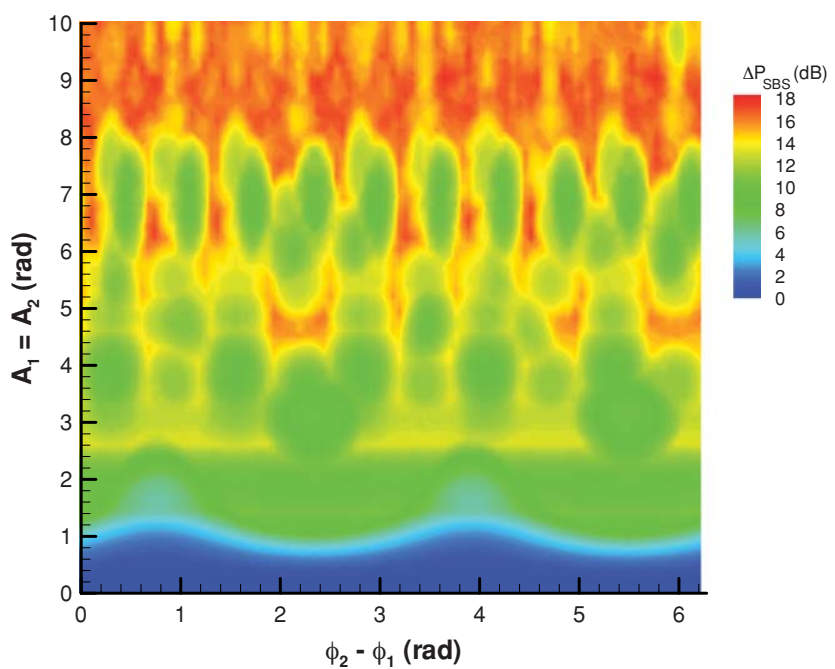

(b)

Fig. 2 Contour plots showing the enhancement in SBS threshold when two tones $(2.5$ and $3 \mathrm{GHz}$ ) are used. The phase difference between the two tones is on the $x$ axis, and the amplitude of the $3-\mathrm{GHz}$ tone is on the $y$ axis. Whereas in (a) the amplitude of the $2.5-\mathrm{GHz}$ tone has been set to unity, in (b) the amplitude of the $2.5-\mathrm{GHz}$ tone is always equal to that of the $3-\mathrm{GHz}$ tone.

Given an optical field with multitone phase modulation, as in Eq. (1), we calculate the SBS threshold power by integrating the total optical power spectral density over the SBS gain bandwidth $\Delta f_{\text {SBS }}$ to obtain the power spectrum as a function of frequency,

$\tilde{P}(f)=\int_{f}^{f+\Delta f_{\mathrm{SBS}}}\left|\tilde{E}\left(f^{\prime}\right)\right|^{2} d f^{\prime}$.

The SBS gain bandwidth depends on the fiber type and can be as large as $100 \mathrm{MHz}$ in the $1550-\mathrm{nm}$ spectral region. ${ }^{11}$ Since we calculate the increase in SBS threshold with respect to the case without phase modulation, we must analogously calculate the power spectrum of the signal without any type of phase modulation,

$\tilde{P}_{0}(f)=\int_{f}^{f+\Delta f_{\mathrm{SBS}}}\left|\tilde{E}_{0}\left(f^{\prime}\right)\right|^{2} d f^{\prime}$.

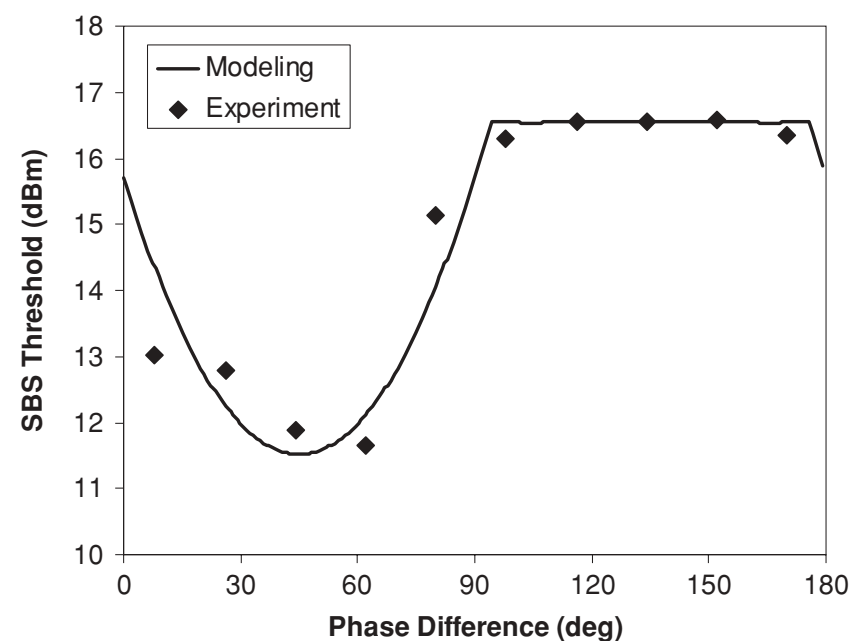

(a)

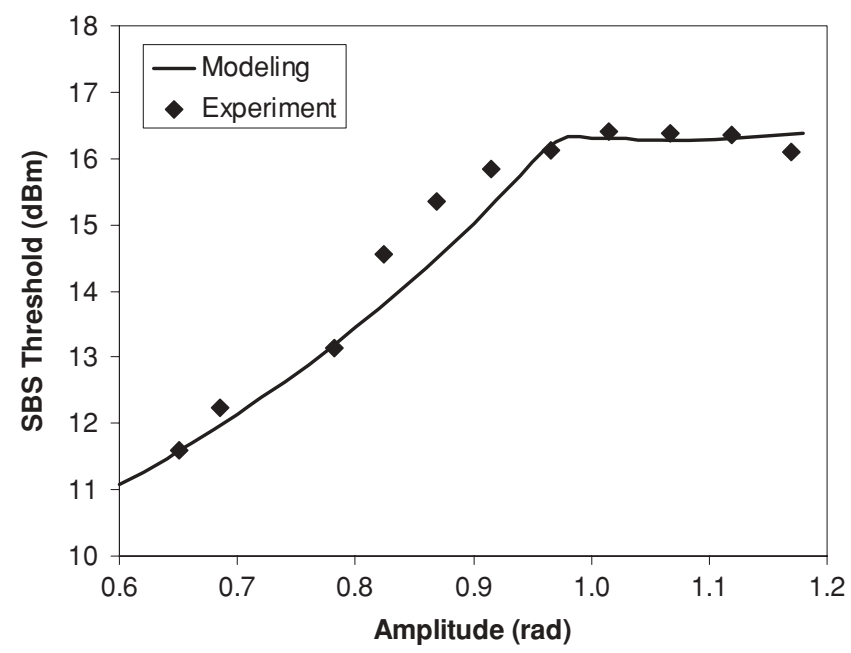

(b)

Fig. 3 Experimental validation of SBS threshold for the case of twofrequency phase modulation: (a) as a function of phase difference and (b) as a function of phase modulator amplitude.

The increase in SBS threshold is then obtained by calculating the maximum value of Eq. (2) normalized to Eq. (3),

$\Delta P_{\mathrm{SBS}}^{t h}=-10 \log _{10}\left(\frac{\tilde{P}^{\max }}{\tilde{P}_{0}^{\max }}\right)$.

Please note that this calculation gives a relative increase in the SBS threshold due to phase modulation and not the absolute value of the SBS threshold power. The denominator, in this case, refers to the SBS threshold without any type of phase modulation.

Using two tones, $f_{1}$ and $f_{2}$, we show the impact that the phase differences between the tones can have on the SBS threshold. We denote the transmitted optical field by

$$
\begin{aligned}
E(t)= & E_{0} \exp \left[i A_{1} \cos \left(2 \pi f_{1} t+\phi_{1}\right)\right] \\
& \times \exp \left[i A_{2} \cos \left(2 \pi f_{2} t+\phi_{2}\right)\right] \\
= & E_{0} \exp \left[i A_{1} \cos \left(\phi_{1}\right) \cos \left(2 \pi f_{1} t\right)\right. \\
& \left.-i A_{1} \sin \left(\phi_{1}\right) \sin \left(2 \pi f_{1} t\right)\right]
\end{aligned}
$$




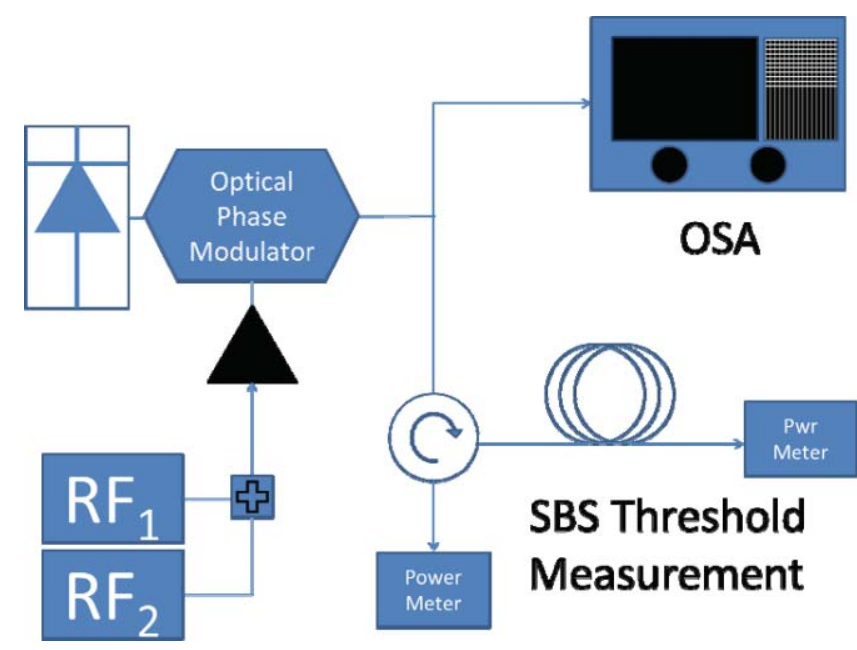

Fig. 4 Setup for the validation experiments.

$$
\begin{aligned}
& \times \exp \left[i A_{2} \cos \left(\phi_{2}\right) \cos \left(2 \pi f_{2} t\right)\right. \\
& \left.-i A_{2} \sin \left(\phi_{2}\right) \sin \left(2 \pi f_{2} t\right)\right] .
\end{aligned}
$$

Since the distribution of the optical spectrum depends not only on the strengths of the phase modulation tones $A_{1,2}$ but also on the phase difference between the tones $\phi_{2}-\phi_{1}$, it is clear that this relative phase can have an important effect on the SBS threshold.

In Fig. 2(a) we show a contour plot of the enhancement in SBS threshold as a function of the amplitude $A_{2}$ and phase $\phi_{2}$ of the second tone, and setting the amplitude $A_{1}=1 \mathrm{rad}$ and phase $\phi_{1}=0 \mathrm{rad}$ of the first tone. We assume $f_{1}=2.5 \mathrm{GHz}$ and $f_{2}=3.0 \mathrm{GHz}$. This contour plot clearly shows that by proper choice of phase difference between the two tones, one can significantly increase the SBS threshold compared to a randomly selected phase difference. Please note that it is often standard practice to set the strength of the second tone to be around $2.4 \mathrm{rad}$ (corresponding to the root of the zero-order $J_{0}$ Bessel function), because around this value the increase in SBS threshold is relatively insensitive to phase. However, we see that by locking the phases properly, even modest increases in the value of $A_{2}$ can lead to substantial increases in the SBS threshold.

Figure 2(b) shows a similar contour plot for $A_{1}=A_{2}$ on the $y$ axis and the phase difference $\phi_{2}-\phi_{1}$ on the $x$ axis. Again, we have $f_{1}=2.5 \mathrm{GHz}$ and $f_{2}=3.0 \mathrm{GHz}$. This contour plot also shows the significance of the relative phase between the two tones. Here, we see that if we combine phase locking with equal drive amplitudes, the SBS threshold can be increased by $17 \mathrm{~dB}$ using $A_{1}=A_{2}$ less than $5 \mathrm{rad}$.

Figure 3(a) shows validation of SBS threshold calculation as a function of phase difference for the case of two-frequency phase modulation, assuming $f_{1}=2.5 \mathrm{GHz}, f_{2}=3.0 \mathrm{GHz}$, and $A_{1}=A_{2}=1.2 \mathrm{rad}$. Agreement between modeling and experiment is very good in both cases. Here the experimental measurements were made using the experimental scheme depicted in Fig. 4. The output of a narrow line-width laser was coupled into a $10-\mathrm{GHz}$ electro-optic phase modulator that was electrically driven with two independent rf sources, paired via a $10-\mathrm{GHz}$ rf amplifier. While the rf signals were driven at the same amplitude $A_{1}=A_{2}=A$, the frequencies $f_{1}=2.5 \mathrm{GHz}$ and $f_{2}=3.0$ were chosen in accordance with Eq. (5), but optimized based on Fig. 2(b) to yield an additional $17 \mathrm{~dB}$ of SBS suppression. The dual-tone, phase modulated optical signal was then launched into subsequent stages to measure the optical spectra and relative SBS threshold with respect to an unmodulated optical signal. Figure 3(b) shows validation for the same setup, but as a function of amplitude, assuming a fixed phase difference of $120 \mathrm{deg}$.

In conclusion, we show that phase locking between the multiple tones of a multitone phase modulator can substantially increase the SBS threshold. This result is of particular value in optical networks requiring high launch powers.

\section{References}

1. A. Kobyakov, S. Kumar, D. Chowdhury, A. B. Ruffin, M Sauer, S. Bickham, and R. Mishra, "Design concept for optical fibers with enhanced SBS threshold," Opt. Exp. 13, 5338-5346 (2005).

2. S. Gray, D. T. Walton, X. Chen, J. Wang, M. J. Li, A. Liu, A. B. Ruffin, J. A. Demeritt, and L. A. Zenteno, "Optical fibers with tailored acoustic speed profiles for suppressing stimulated Brillouin scattering in high-power, single-frequency sources," IEEE J. Select. Top. Quant. Electron. 15, 37-46 (2009).

3. S. Mussot, M. Le Parquier, and P. Szriftgiser, "Thermal noise for SBS suppression in fiber optical parametric amplifiers," Opt. Commun. 283, 2607-2610 (2010).

4. V. I. Kovalev and R. G. Harrison, "Suppression of stimulated Brillouin scattering in high-power single-frequency fiber amplifiers," Opt. Lett. 31, 161-163 (2006).

5. C. Wang, F. Zhang, Z. Tong, T. Ning, and S. Jian, "Suppression of stimulated Brillouin scattering in high-power single-frequency multicore fiber amplifiers," Opt. Fiber Technol. 14, 328-338 (2008).

6. F. W. Willems, J. C. van der Plaats, and W. Muys, "Harmonic distortion caused by stimulated Brillouin scattering suppression in externally modulated lightwave AM-CATV systems," Electron. Lett. 30, 343-345 (1994).

7. S. L. Woodward and T. E. Darcie, "A method for calculating multipath interference noise," Photon. Tech. Lett. 6, 450-452 (1994).

8. F. W. Willems and W. Muys, "Suppression of interferometric noise in externally modulated lightwave AM-CATV systems by phase modulation," Electron. Lett. 29, 2062-2063 (1993).

9. F. W. Willems, W. Muys, and J. S. Leong, "Simultaneous suppression of stimulated Brillouin scattering and interferometric noise in externally modulated lightwave AM-SCM systems," Photon. Technol. Lett. 6, 1476-1478 (1994).

10. J. B. Coles, B. P. P. Kuo, N. Alic, S. Moro, C. S. Bres, J. M. Chavez Boggio, P. A. Andrekson, M. Karlsson, and S. Radic, "Bandwidthefficient phase modulation techniques for stimulated Brillouin scattering suppression in fiber optic parametric amplifiers," Opt. Express 18, 18138-18150 (2010).

11. G. P. Agrawal, Nonlinear Fiber Optics, 3rd ed., p. 359, Academic, New York (2001). 